\title{
Singing Cans
}

\section{Prototyping an experimental wind instrument through parametric design integrated with field experiments}

\author{
Shao-Yun Wang ${ }^{1}$, Agathi Sianoudi ${ }^{2}$, Maohua Wang $^{3}$, Hongmei Wu ${ }^{4}$, \\ Tsung-Hsien Wang ${ }^{5}$, Zhuoqun Zhang ${ }^{6}$, Chengzhi Peng ${ }^{7}$ \\ 1,2,3,4,5,7 School of Architecture, University of Sheffield ${ }^{6}$ Department of Electrical \& \\ Electronic Engineering, University of Sheffield \\ 1,2,3,6 \{stella6151|agathi.sianoudi|maohuawang94|blockdiechmann\}@gmail. \\ com4hwu923@yeah.net ${ }^{5,7}\{$ tsung-hsien.wang|c.peng\}@sheffield.ac.uk
}

We present a study of how parametric design can be linked to field experiments where ready-made plug-ins are not available for performative modelling. The study centres on prototyping 'Singing Cans' - an experimental wind instrument made with an assembly of drinking cans that can produce sounds in recognizable pitches by interacting with airflows. We describe how field experiments conducted in a fluid flow lab can generate performative resources linkable to parametric design modelling. In Singing Cans, we focus on how to get airflow through a hole made on drinking can to make sounds. The prototyping process involved a lab-based calibration process to establish the relationship between the air volume of a can, measured by water-filling, and the pitch produced, measured by the Tuner Lite by Piascore. The field experiments resulted in a dataset capturing a can's sound-making behaviour in terms of water volumes and pitches. A parametric model that can take in wind data generated by a CFD package and output a 3D frame for site-specific cans installation is presented.

Keywords: parametric design, field experiments, experimental wind instrument, fluid flow instrumentation, sound production

\section{RESEARCH CONTEXT: SITE-SPECIFIC UR- BAN WIND ENVIRONMENTS}

We propose an idea of urban musical instrument that originates from urban wind environments.

The urban wind flows are seen as a sound-making system that drives an array of disused drinking cans. We consider the idea in an urban context where the wind flows exhibit various patterns shaped by different building densities. As shown in Figure 1, the higher aspect ratio (H/W) can create a stable circulatory vortex between buildings (Oke 1988).

Therefore, we are interested in looking for narrow streets as potential installation sites. The design challenge here is how to develop a generative system for configuring recognisable pitches generated by wind 
flows for people to hear and interact - Singing Cans.

In general, the principle of sound production is related to the vibration and movement of the air molecules in the surrounding area. For instance, in flute playing, the lips direct the air stream against the thin edge of the mouthpiece, raising the resonance of the pipe to produce a tone (Farnol 1941). A general name of the instruments that produce sounds through fluid dynamics is called reustophones (Mann and Janzen 2015).
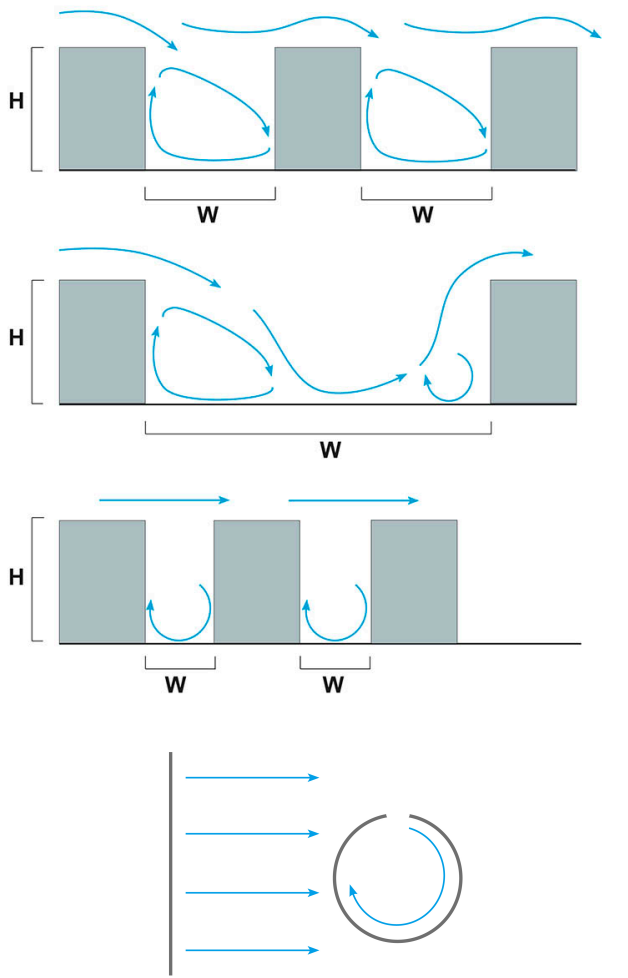

In our project, the design of a frame is required to assemble Singing Cans in a site-specific way and maintains the same wind pattern after installation. By fol- lowing the flow regimes, parametric frames are created and placed in a specific location with the extract wind data from the CFD simulation.

In Singing Cans, we focus on how to get airflow through a hole made on the cans to produce sounds. Aluminum cans were selected as the main form and material because it was easy to find them from recycling points. To assemble the cans into a site-specific installation, we focus on how to get the wind data from the site and then use them to generate a frame. This paper shows (1) how we catch the airflow to produce sounds following the principle of the music production of the flute; (2) how we create the installation frame based on CFD simulation of urban microclimate, following the adaptive parameters in terms of the size of the frame and cans.

\section{SOUND-MAKING CANS}

From a basic study of sound-making with cans, we moved to generate pitches from cans. This involved a lab-based calibration process to establish the relationship between the air volume of a can, measured by water-filling, and the pitch produced, measured by the Tuner Lite app by Piascore. The field experiments resulted in a dataset capturing a can's sound-making behaviour in terms of water volumes and pitches. When the wind blows through a pipe, it does not produce sounds all the time; only when the air is resonating in the chamber, then noises could be heard (Benade and French 1965). The phenomenon can be further explained by Reynolds number in fluid dynamics.

It was found that the relationship of the fluid density of air, the flow velocity and the diameter of a pipe influences turbulence flows in the pipe (Upp and LaNasa 2002). A pipe sounds when the Reynolds number reaches a certain level, and sounds could be heard as the air inside the pipe is vibrant and resonate. In Singing Cans, we found that the angle of airflow into a can is an important factor. The airflow going through the sharp edge cut open on a can becomes turbulent from a laminar state (Raman and Srinivasan 2009). In order to identify certain valid an-
Figure 1

The wind flows characterised in the building-street aspect $(\mathrm{H} / \mathrm{W})$ ratios (after Oke 1988)

Figure 2

Wind flows through a can with a sharp edge cut open 
Figure 3 Wind testing of cans in the Lab

Figure 4

Pitches in Cans

Figure 5

Sound production with the cans gles of the input airflow, we created a hole in each can and we tested them by rotating the cans in different directions.
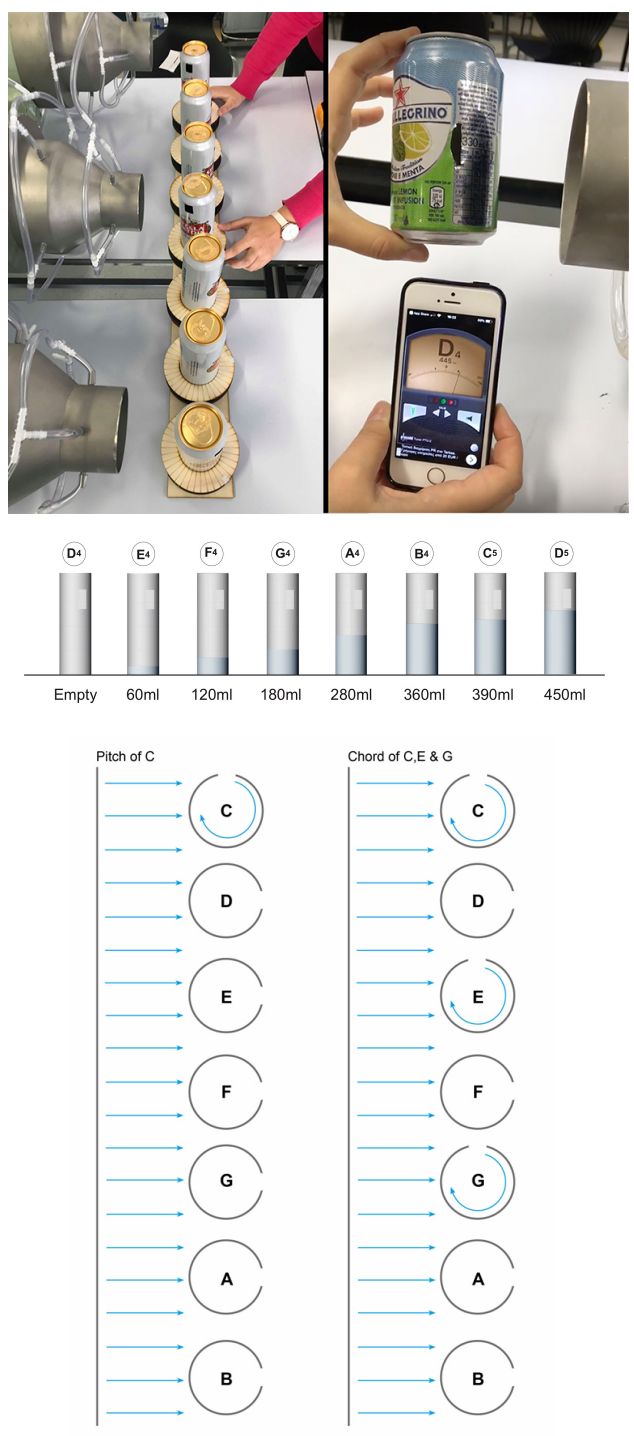

Our lab experiments show that the cans produced sounds within a particular range of 60 to 90 degree. Throughout the testing, we concluded that the can should be tangent to the airflow as indicated in Figure 2 .

In the lab, a wind tunnel (Figure 3) was used to measure the pitches produced by the cans. In the beginning, a single can of $330 \mathrm{ml}$ was tested. We first noticed that a certain angle of the hole in relation to the airflow, around 90 degrees (Figure 2), was required for the can to sound optimal. As the wind tunnel can be controlled to produce different flow speeds, we investigated the measurement of pitches in 445 Hertz frequency level. When the wind speed was high enough, the result was a louder sound with no stability and sometimes the pitch was converting into noise. Therefore, the wind speed was stabilised at approximately $7.5 \mathrm{~m} / \mathrm{s}$ for taking the measurements as the sound data.

Our laboratory experiments provided useful numerical data and knowledge on the conditions of how a can produces different pitches. Cans with different sizes were further tested. The observation was that different sizes produced different pitch readings. As expected, cans of fewer air volumes resulted in higher pitches. In order to obtain a more accurate pitch measurement, water was used to control the air volume inside the can. To produce a wider range of notes, a can of the $568 \mathrm{ml}$ volume was tested. Eight cans of the same volume size, filled with different volumes of water inside, were tested separately to produce one note. A range of eight musical notes (D4, E4, F4, G4, A4, B4, C5 and D5) was then generated into a dataset (Figure 4). Subsequently, we control the rotation of the cans to produce seven pitches or the chord of pitches like Figure 5. Each can was programmed to be on or off for a certain duration to create the melody.

\section{SENSING CURRENT WIND DATA}

For further development, the ranges of airflow velocity and direction were considered as the essential input data to a generative sound-making system. 


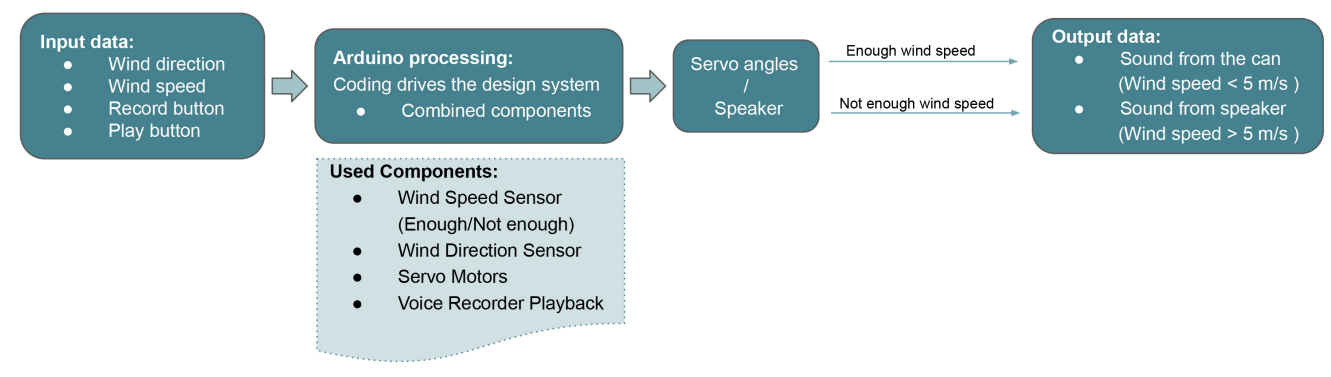

Table 1: Arduino components

\begin{tabular}{ccc}
\hline & \multicolumn{1}{c}{ Input } & Output \\
\hline \multirow{2}{*}{$\begin{array}{c}\text { Arduino } \\
\text { Sensors } / \\
\text { components }\end{array}$} & 1 Anemometer (Wind Speed) & 1 Relay \\
\cline { 2 - 3 } & $\begin{array}{l}\text { Additional components: 1 Mega 2560 board, 1 big breadboard, 1 small breadboard, 1 power supply, Resistors } \\
\text { and Jumper wires. }\end{array}$ & 2 push buttons \\
\cline { 2 - 3 } & & 1 ISD1820 Voice Recorder Playback \\
\hline
\end{tabular}
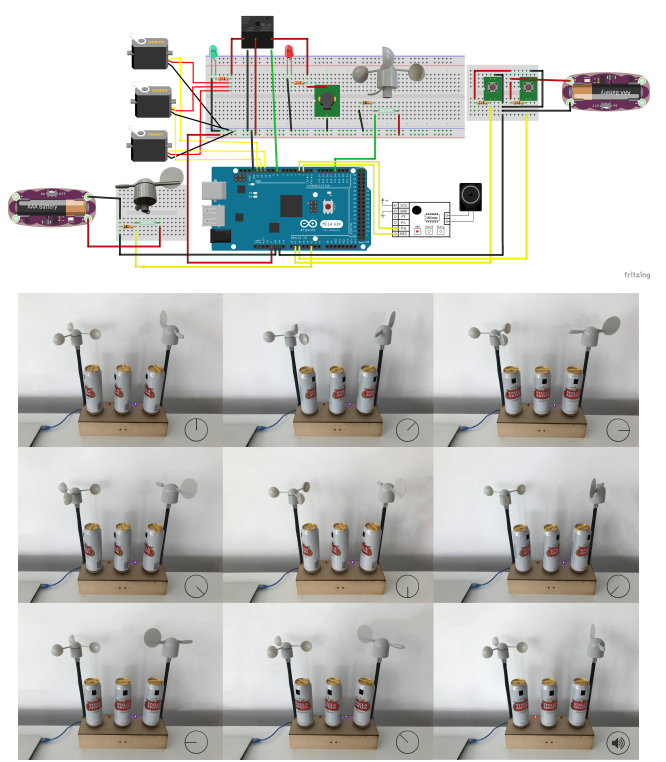

Arduino is a useful resource for us to construct a wind sensing platform. The application method comes from an anemometer and a wind vane. The process shown in Figure 6 starts with the current wind speed and wind direction as the input data to the Arduino system. The microcontroller activates servo motors to rotate the cans following the angle reading from wind vane direction. We also develop an alternative method: If the wind velocity is not high enough for the cans to produce audible sounds, a speaker will be activated to play pre-recorded sounds instead.

In prototyping the interactive Singing Cans installation, we have utilised a number of physical computing components (Table 1). These components are divided into input and output groups. Figure 7 shows how the system works. The process begins with the input data of the wind direction and the wind speed. Two push buttons were used for sound recording. When processing these data, the system makes two decisions based on the inputs (Figure 8): (1) if a wind
Figure 6

An Arduino-based wind sensing platform

Table 1

Arduino components

Figure 7

Arduino assembly diagram

Figure 8

Sensing wind direction to rotate the cans 
Figure 9

Extract wind data from Envi-met

Figure 10

The formula for calculating the new vector

Figure 11

The way to define the boundary
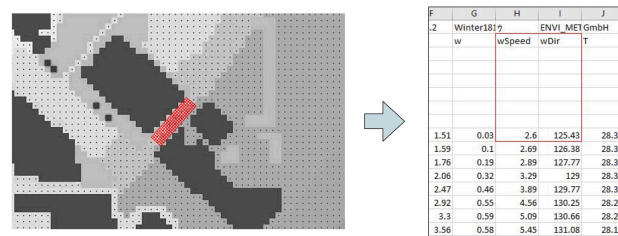

speed of $5 \mathrm{~m} / \mathrm{s}$ is reached, the cans will be rotated to align with the wind direction. As such, the cans will be able to generate sound from the airflow; (2) if a wind speed below $5 \mathrm{~m} / \mathrm{s}$ is measured, then the speaker is activated to play the recorded sound file.
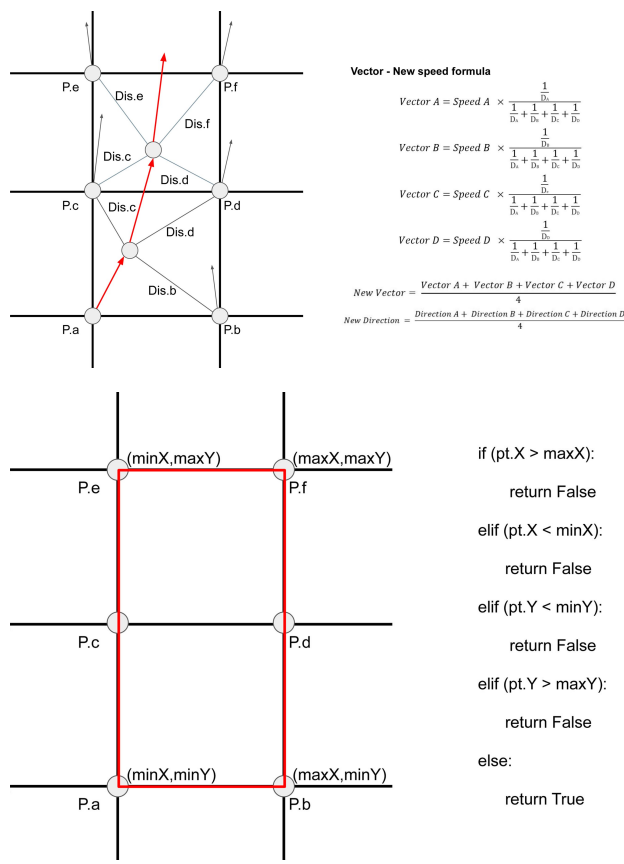

\section{PARAMETRIC MODELLING OF THE FRAME} DESIGN

In order to determine the geometry of a frame design for site-specific installation, we first used ENVI_MET (a CFD urban climate modelling package) to generate wind data. The simulated wind data were then imported to a parametric modelling process using Rhino-Grasshopper (Figure 9). The data comes from ENVI_MET software which investigates CFD simulation in climatological conditions [1]. Then, a uniform rectangular grid with the spacing of 1 meter along the $x$ - and $y$-axis was used for both modelling and simulating in ENVI-met. Each grid point was the observation point of wind data for the speed and direction. Finally, the simulation results were extracted into spreadsheets for following windinformed parametric modelling design in Rhinoceros 3D and Grasshopper [2].

With the initial grid-based wind conditions, data interpolation was implemented with the inverse distance relationship to the existing grid points. In the 3D modelling environment, wind data is represented by the vector. The wind speed defines the length of the vector and the wind direction defines the angle of the vector. There are two customised procedures implemented in Python within the Rhino-Grasshopper scripting environment [3]. The first is to generate a parametric curve following the wind direction and speed. We recursively define new vectors with the closest four neighbours while moving forward. The second step is to determine the termination criteria given the desired boundary conditions. 

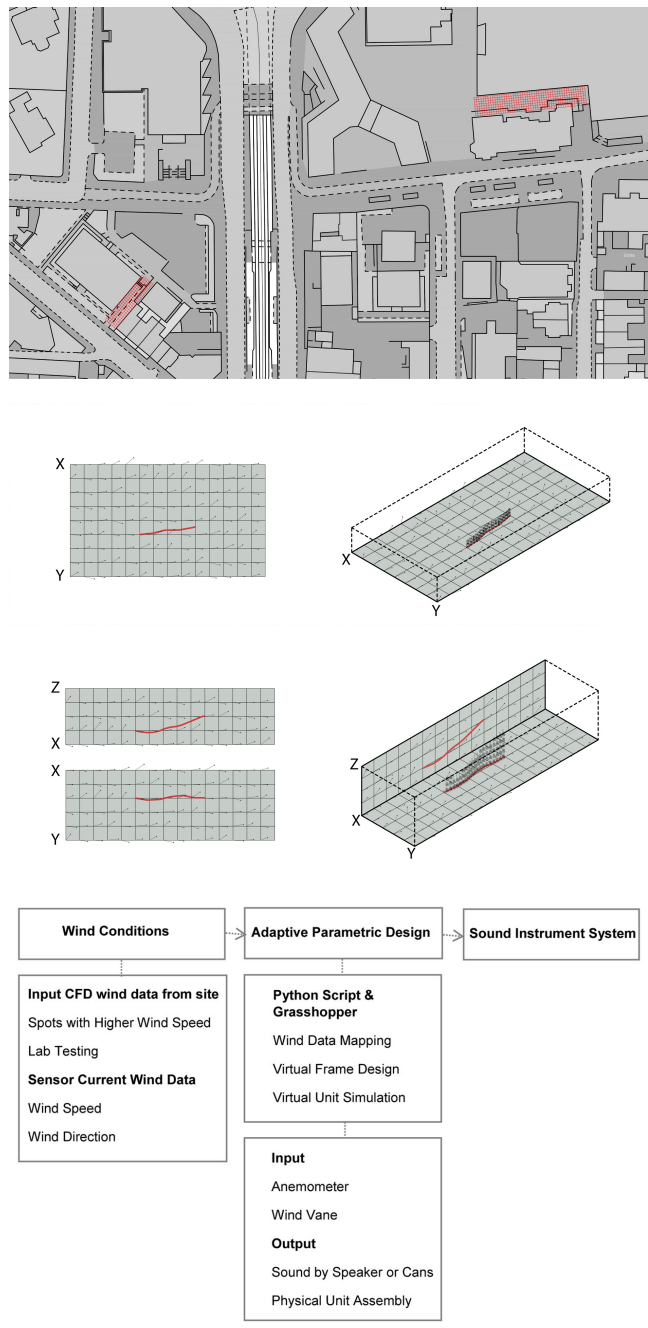

The inverse distance formula is used to calculate the distance relationships from a target location with four closest neighbour grid-points. These relationships are later employed to define new interpolated wind direction to move the target point forward with a unit length, as shown in Figure 10. This step is executed recursively until the termination crite- ria are met. By specifying the desired boundary as showed in Figure 11, the termination procedure checks whether the new target location exceeds the set boundary. If true, the curve generation will be terminated.

\section{THE SITE ADAPTATION PROCESS}

Based on specific wind data, discovering the ideal architectural shape can be realized by parametric modelling (Kormaníková et al. 2018). We adopted a computational fluid dynamics (CFD) site modelling approach to the frame design. For meeting the design and environmental requirements, the chosen site needs to contain two conditions: (1) to select the city corner as the application scene; (2) to obtain a relatively stable high wind velocity environment, the site needs to present a narrow and long corridor space. Considering the above considerations, two locations on the University of Sheffield campus were selected to collect wind data and generate structural forms (Figure 12).

Due to the need for adapting to different site characteristics, here two different vertical and horizontal structures were proposed. Site 1 provides a wall at a height of approximately $1100 \mathrm{~mm}$, and the installation can be placed directly above this wall, so a horizontal frame is designed (Figure 13). At site 2, the installation is directly placed on the ground, and the frame structure needs to have a specific height. The vertical height of the frame is generated by the $X Z$ section of wind data to determine the height of each column, so a vertical framework is shown in Figure 14.

\section{ON-SITE DISPLAY OF URBAN MUSICAL IN- STRUMENT}

The development of 'Singing Cans' installation combines virtual and physical prototyping to test how digital tools can successfully activate the interactive features (Figure 15). In this project, we start by identifying locations with higher wind speeds on campus to investigate on-site wind conditions through simulation. We then incorporate the simulated data to
Figure 12

Two locations on the University of Sheffield campus selected for Singing Cans installation

Figure 13

A horizontal frame

Figure 14

A vertical frame

Figure 15

The overall workflow of the Singing Cans prototyping 
Figure 16

A horizontal frame rendering on site formulate the wind flows into parametric shapes for the structure frame design. The proposed frame de-

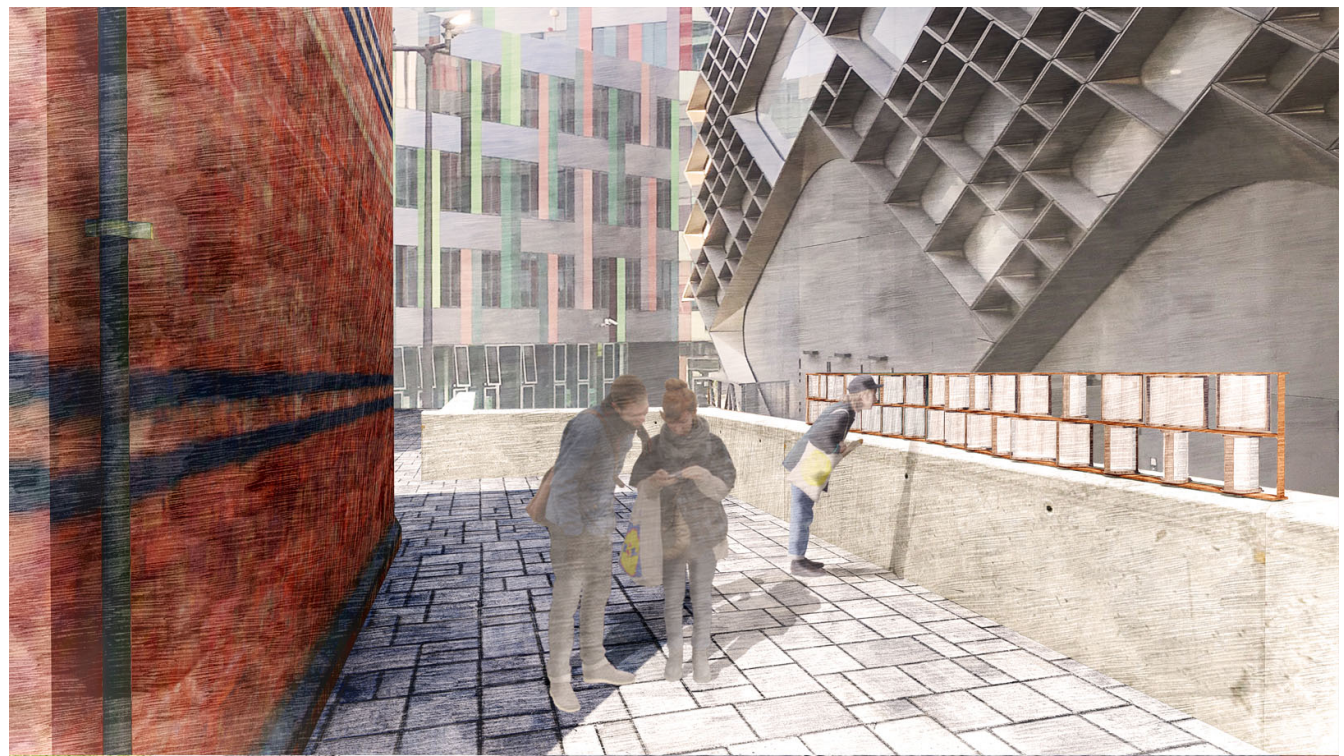

Figure 17

A vertical frame rendering on site

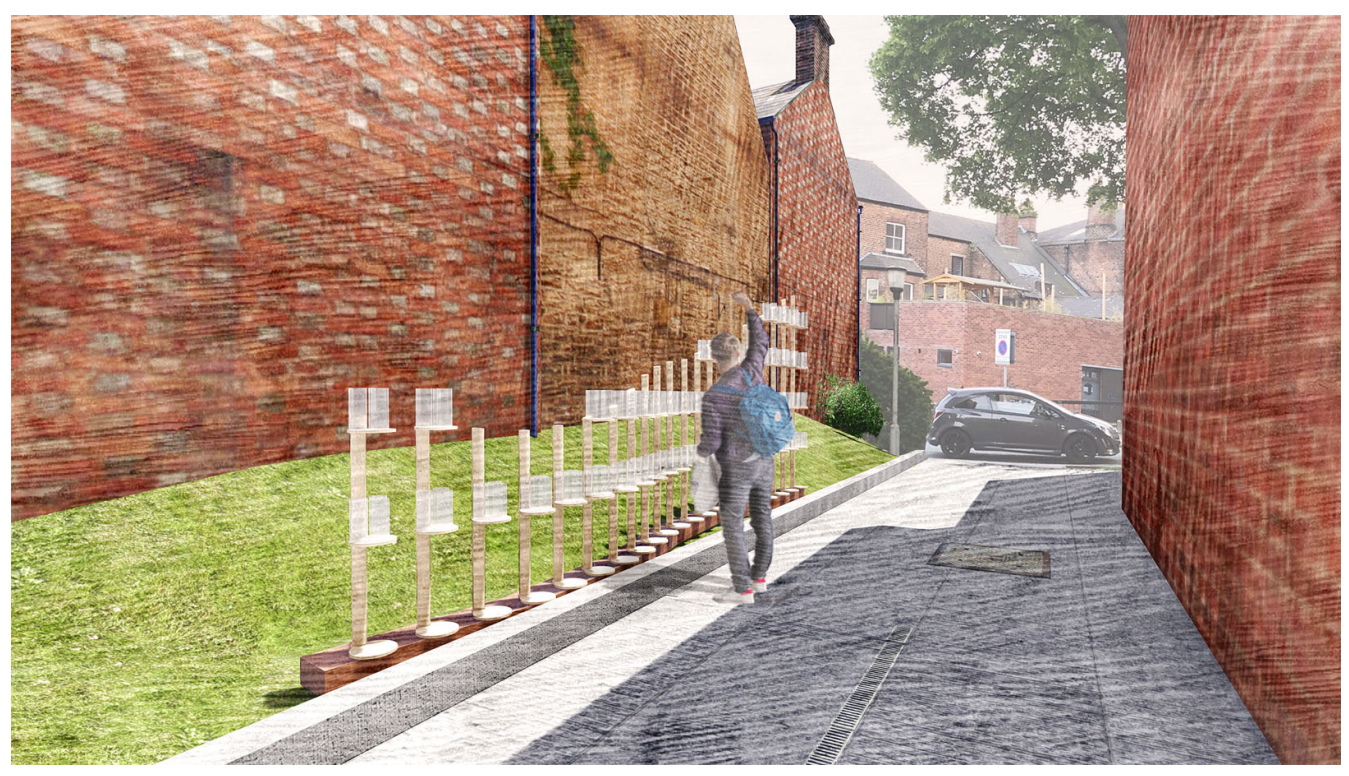


sign is envisaged to receive enough wind speed and not to interfere with the existing wind conditions on site.

\section{CONCLUSION AND FURTHER DEVELOP- MENT}

The Singing Cans urban wind instrument can be further improved, by implemented alternative rotation methods to generate different sounds of various volumes in the cans and use more push buttons to switch the rotating patterns of them. For further research, an Internet of Thing (IoT) user interface could be developed to extend the interaction design. The IoT layer will link up multiple installations at different sites (Figure 16 and Figure 17). The mobile device based application can display real-time wind data for the users as hints to navigate through the city's wind environments. Thus, the Singing Cans urban musical instrument not only provides a system for the public to interact with the wind and sound environments but also offers a practical facility to collect wind data.

\section{ACKNOWLEDGEMENTS}

We thank Krys Bangert who provided us with a space to work in the Fluid Dynamics Lab at the University of Sheffield for testing the cans, and also Binh Vinh Duc Nguyen who advised us at the early stages of this project.

\section{REFERENCES}

Benade, AH and French, JW 1965, 'Analysis of the Flute Head Joint', The Journal of the Acoustical Society of America, 37, p. 679

Farnol, HH 1941, 'Physiology Applied to Music', Music Educators Journal, 27(4), p. 17-73

Kormaníková, L, Achten, H, Kopřiva, M and Kmet', S 2018, 'Parametric wind design', Frontiers of Architectural Research, 7(3), pp. 383-394

Mann, S and Janzen, R 2015, October 'Fluid input devices and fluid dynamics-based human-machine interaction', 2015 IEEE Games Entertainment Media Conference (GEM), pp. 1-4

Oke, TR 1988, 'Street design and urban canopy layer climate', Energy and Buildings, 11(1), p. 103-113
Raman, G and Srinivasan, K 2009, 'The powered resonance tube: From Hartmann', Progress in Aerospace Sciences, 45, pp. 97-123

Upp, EL and LaNasa, PJ 2002, Fluid Flow Measurement: A Practical Guide to Accurate Flow Measurement, Elsevier

[1] https://www.envi-met.com/windandsun/

[2] https://www.grasshopper3d.com/group/ghowl

[3] https://developer.rhino3d.com/api/RhinoScriptSynt ax/ 